\title{
RESOLVE OVEN Field Demonstration Unit for Lunar Resource Extraction
}

\author{
Aaron Paz ${ }^{1}$, Lara Oryshchyn ${ }^{1}$, Scott Jensen ${ }^{1}$, Gerald B Sanders ${ }^{1}$, Kris Lee $^{1}$ and Mike Reddington ${ }^{2}$ \\ NASA Johnson Space Center, Houston, TX, 77058
}

\begin{abstract}
The Oxygen and Volatile Extraction Node (OVEN) is a subsystem within the Regolith \& Environment Science and Oxygen \& Lunar Volatile Extraction (RESOLVE) project. The purpose of the OVEN subsystem is to release volatiles from lunar regolith and extract oxygen by means of a hydrogen reduction reaction. The complete process includes receiving, weighing, sealing, heating, and disposing of core sample segments while transferring all gaseous contents to the Lunar Advanced Volatile Analysis (LAVA) subsystem. This document will discuss the design and performance of the OVEN Field Demonstration Unit (FDU), which participated in the 2012 RESOLVE field demonstration.
\end{abstract}

\section{Introduction}

$\mathrm{T}$ HE ability to utilize resources found in-situ has historically been critical to the success of human exploration on Earth. NASA recognizes that this may also be true for the future of human space exploration. The identification of water on other worlds has been an objective of several NASA missions. The presence of water ice on Mars has now been confirmed by the Phoenix mission ${ }^{1}$. Similarly, evidence of water ice on the Moon has been detected by the Lunar Crater Observation and Sensing Satellite (LCROSS) and Lunar Reconnaissance Orbiter (LRO) ${ }^{2}$. Now that water is known to exist on the Moon and Mars, we can begin to think about how this water can be located, mapped, extracted, and utilized for the benefit of human exploration.

NASA and the Canadian Space Agency (CSA) are currently in the early stages of developing hardware that will attempt to extract water ice near the south pole of the moon, as well as collect data on the distribution, depth, and concentration of volatiles. The Regolith and Environment Science \& Oxygen and Lunar Volatile Extraction (RESOLVE) is a rover-based payload aimed at answering questions about the type, amount, and location of possible resources at the lunar poles, especially water/ice, as well as demonstrate the extraction of oxygen from lunar regolith $^{3}$. To meet these mission objectives, the RESOLVE payload consists of several subsystems to locate hydrogen rich volatiles below the surface, acquire and analyze subsurface samples, extract and measure volatiles present in the sample, and capture water released from the sample and from the oxygen extraction process. To locate hydrogen rich volatiles, RESOLVE utilizes a neutron spectrometer (NS) and Near Infrared (NIR) spectrometer. The NS can measure hydrogen down to $\sim 1$ meter below the surface and the NIR spectrometer can measure water and hydroxyl molecules on the surface of regolith examined. Once a location of interest has been identified, RESOLVE utilizes a sample acquisition system (SAS) provided by the CSA. The SAS incorporates an auger to perform a quick evaluation of regolith mechanics while bringing material to the surface (down to $\sim 50 \mathrm{~cm}$ ) for evaluation by the NIR spectrometer, a 1 meter push tube, and a 1 meter coring drill. Depending on whether the regolith is unconsolidated or consolidated with water/ice, the push tube or coring drill will be used to acquire a full one meter core. The core is extracted and transferred to the Sample Transfer Receptacle (STR) where the sample is divided into segments (one at a time) for evaluation. The sample segment from the STR is transferred to the Oxygen and Volatile Extraction Node (OVEN) subsystem where the sample is heated to $423 \mathrm{~K}$ to evolve any volatiles present. The volatiles released are then sent to the Lunar Advanced Volatile Analysis (LAVA) subsystem where the chemical and isotopic content of the volatiles is measured by a combined mass spectrometer and gas chromatograph (MS/GC). The MS/GC is designed to sense both solar wind volatiles such as hydrogen, carbon, nitrogen, as well as detect compounds such as water, carbon monoxide, methane, or ammonia from comets that might have been trapped in the permanently shadowed regions of the lunar poles. Once the volatile characterization portion of the experiment is complete, the OVEN subsystem can raise the temperature of the sample segment to more than $1173 \mathrm{~K}$ in the

\footnotetext{
${ }^{1}$ Propulsion and Power Division/ Energy Conversion Systems, 2101 Nasa pkwy/Mailcode EP3

${ }^{2}$ Propulsion and Power Division/ Energy Systems Test Area, 2101 Nasa pkwy/Mailcode EP6

*AIAA member
} 
presence of hydrogen $\left(\mathrm{H}_{2}\right)$ gas to extract oxygen from the iron oxides in the lunar regolith. The water released from the volatiles or produced in the hydrogen reduction reaction can be further separated and imaged with the Water Droplet Demonstration in the LAVA subsystem.

In July of 2012, the RESOLVE development team demonstrated the end-to-end functionality of locating, mapping, extracting, and measuring volatiles from subsurface samples during an analog field test on the slope of Mauna Kea in Hawaii. The OVEN subsystem was one of the instruments being evaluated during this analog site demonstration. The overall functionality and performance of the OVEN field demonstration unit (FDU) will be discussed in this document.

\section{Design Overview}

The OVEN subsystem must perform a variety of functions involving gravimetry, mechanical movements, thermal processing and chemical processing that together present a unique design challenge. The first task is to receive a $16 \mathrm{~mm}$ diameter $\mathrm{x} 12.5 \mathrm{~cm}$ regolith core sample segment. The segment can be unconsolidated, solidified with water ice, or any combination of the two. More importantly, the temperature of the segment will likely be less than $200 \mathrm{~K}$ if it contains water ice ${ }^{4}$. Therefore, any container within the OVEN subsystem that receives the segment should have an initial temperature less than that of the segment. Otherwise, a volatile release may occur before the segment is contained within a sealed environment. After receiving a segment, the next task of the OVEN is to weigh it, so that a water/volatile concentration by mass can be determined. A sealed environment can then be created around the segment. As heat is applied to the sealed container, water vapor and other volatiles will create pressure. Gas is transferred to the LAVA subsystem for quantification and analysis until water is no longer being extracted from the segment. An additional process, known as hydrogen reduction, is applied to some segments in order to remove oxygen from the regolith. This proces requires regolith to be heated to approximately $1173 \mathrm{~K}$ in the presence of hydrogen gas. Water vapor is produced from the reduction of iron oxide compounds contained within the regolith $^{5}$. Upon completion of the volatile analysis or oxygen extraction step, the segment is removed from the OVEN.

The field demonstration unit was developed to operate in a laboratory environment, so the OVEN FDU was only required to accept core segments at room temperature. However, it was apparent that the most challenging requirement to an OVEN system operating on the lunar surface will be maintaining the initial segment temperature. Many concepts were considered for the OVEN FDU, but when keeping the lunar environment in mind, the low segment temperature is what ultimately leads to a reusable crucible approach. Multiple crucibles allow for the OVEN to minimize energy consumption with minimum processing time, because heating and cooling can take place simultaneously at separate locations. An overview of the OVEN FDU system is illustrated in Fig. 1.

\begin{tabular}{|c|c|c|c|}
\hline NOMINAL MISSIONLIFE & $\frac{\text { MASS }}{13 \mathrm{~kg}}$ & DIMENSIONS & PEAK POWER \\
\hline 40 segments & $.275 \times 375 \times 335 \mathrm{~mm}$ & $200 \mathrm{~W}$ \\
\hline
\end{tabular}

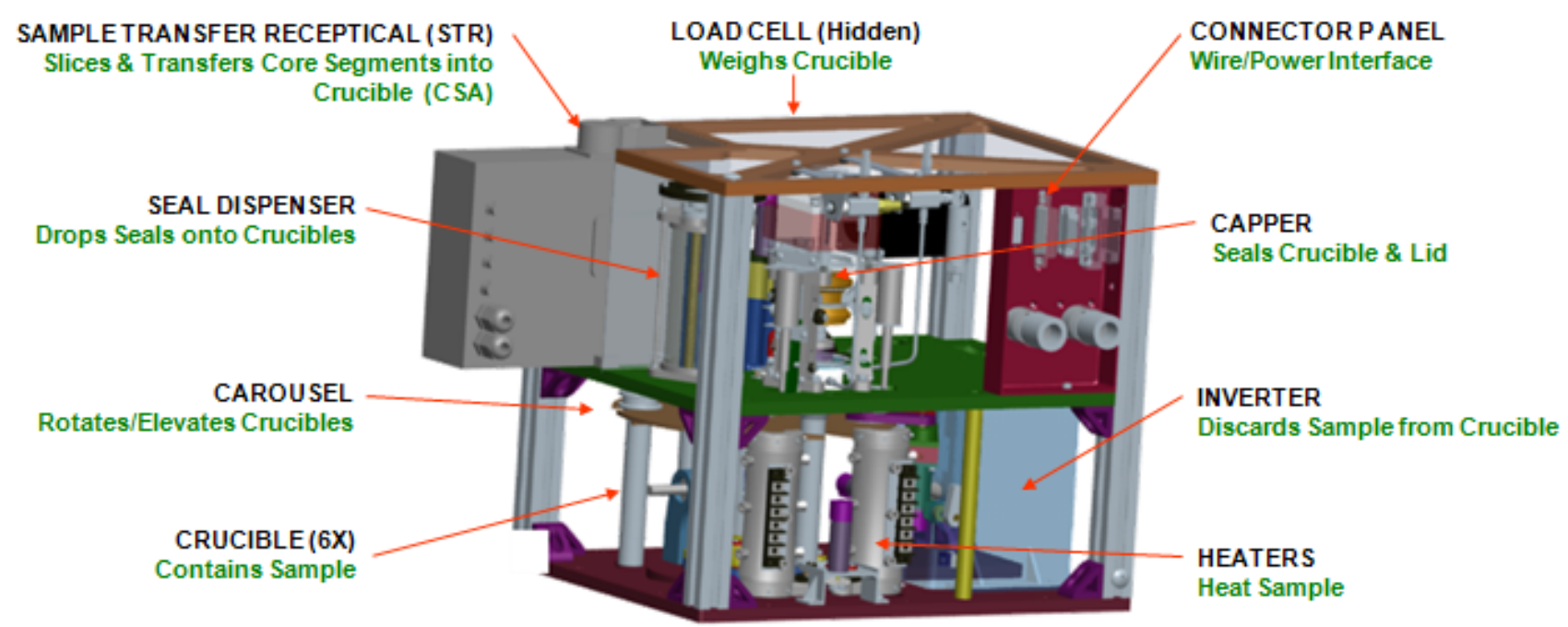

Figure 1. OVEN FDU overview 
The OVEN FDU contains six inconel crucibles placed on a carousel. The carousel can move 360 degrees in either direction as well as change elevation in order engage the crucibles in an array of mechanisms positioned around the system. The mechanisms are evenly spaced 60 degrees apart with stowage stations alternating between them. The components identified in Fig. 2 are further described as follows: Snubbers are used to stow all six crucibles while the rover is moving. Sample segments enter the system through the sample transfer receptacle (STR) provided by the Canadian Space Agency. The weigh station contains a load cell and mechanized claw assembly that enables crucibles and sample segments to be weighed. The seal dispenser can contain up to 50 disposable seals which are placed onto a crucible after a segment has been loaded and weighed. The reactor is the combination of two axially aligned mechanisms reffered to as the capper and clamshell heater. The capper pulls a crucible against a fixed reusable lid in order to create a seal. After a seal is made, the clamshell heater is closed and surrounds the crucible. The inverter is used to remove the segment after volatiles or oxygen have been extracted. Fig. 2 illustrates the segment processing sequence and shows the placement of mechanisms and stowage snubbers.

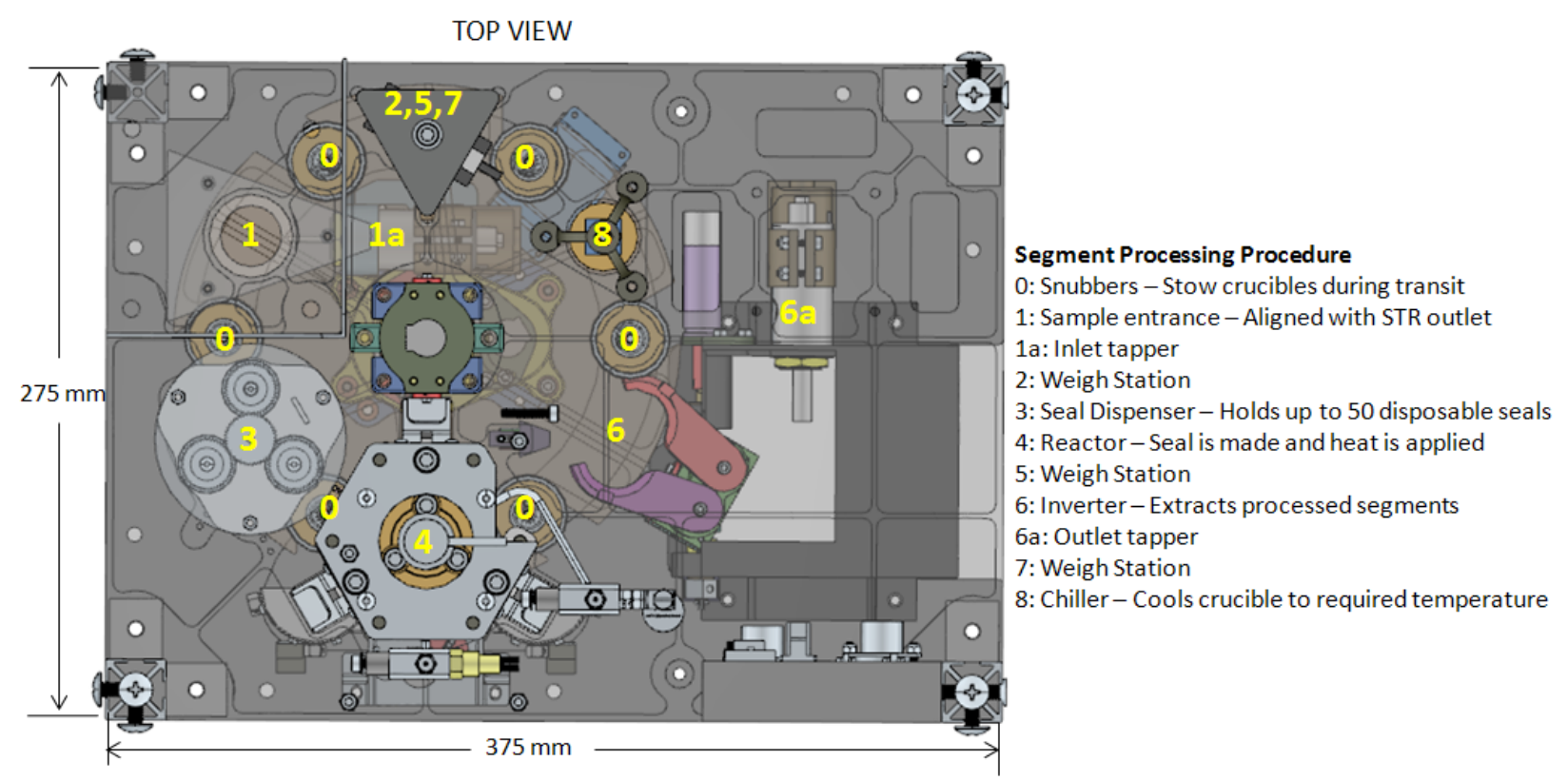

Figure 2. OVEN sequence and mechanism placement

\section{Subassembly Design and Performance}

Like most complex systems, the OVEN is a compilation of several simpler parts. The OVEN system can be broken down into a few basic functions. Each function is addressed by a single subassembly, with only one job to perform. This allows for maximum flexibility in the design process. If one component does not work properly, it can be redesigned, modified, or replaced without impacting other parts of the system. The following sections will discuss the individual design and performance of each subassembly.

\section{A. Structure}

One of the goals of the field demonstration was to expose hardware to dust similar to that found on the lunar surface. For the FDU, the structural boundary is used as a means of dust mitigation. The walls of the OVEN are made of polycarbonate sheets. The frame is made of extruded aluminum with a square cross section. The walls are secured against the aluminum frame and taped at the seams. During the field demonstration, only the upper portion of the OVEN was specifically designed to mitigate dust. The simple method illustrated in Fig. 3a proved to keep dust out. Fig. 3b illustrates the effectiveness of the FDU dust mitigation strategy in the upper portion of the OVEN.

The lower portion of the OVEN FDU was not specifically designed to mitigate dust. This is because core segments are discarded through the bottom plate of the OVEN. Dust was allowed to enter the bottom half of the system, providing an opportunity to observe the extent to which dust enters, and its effect on mechanisms. Fig. 3c illustrates the dust infiltration observed in the lower portion of the OVEN FDU. Although a significant amount of dust entered, the dust did not specifically impact the performance of mechanisms during the field demonstration. 
Regardless, future OVEN design iterations will include a dust hatch at the sample exit to prevent dust from entering the system.

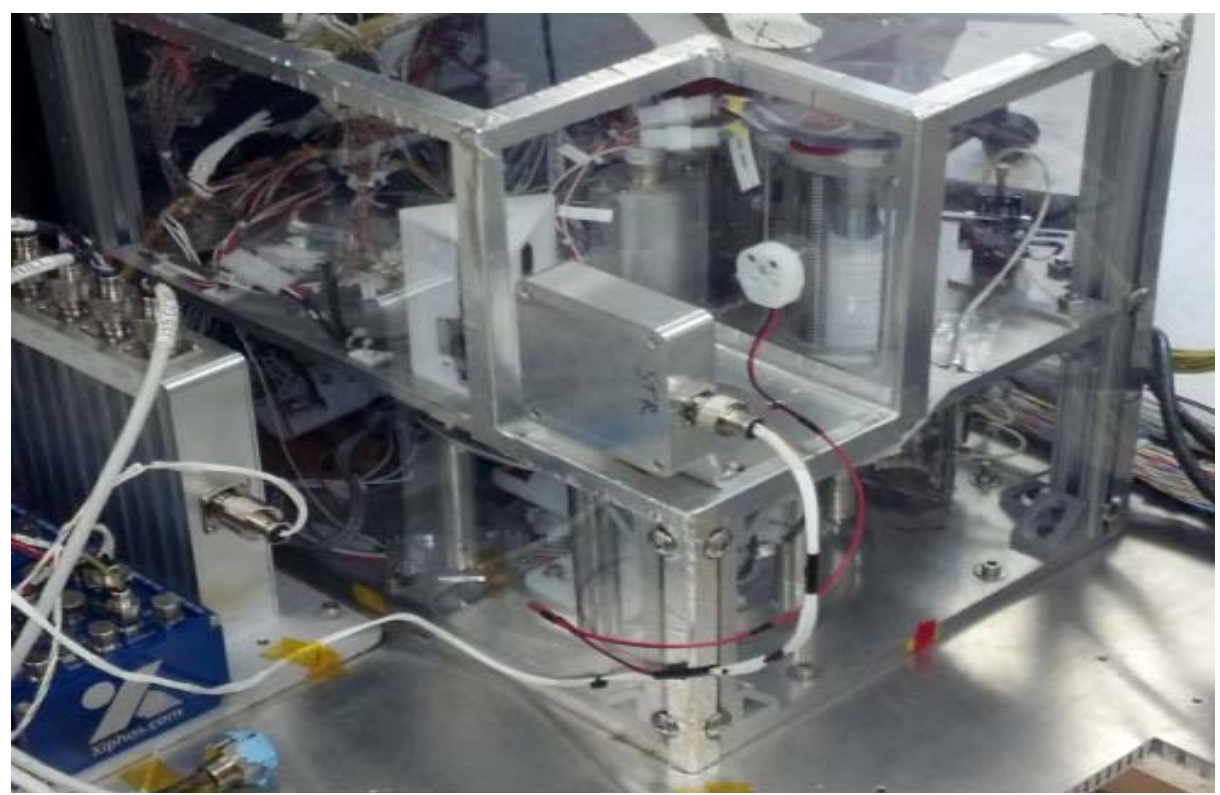

(a)

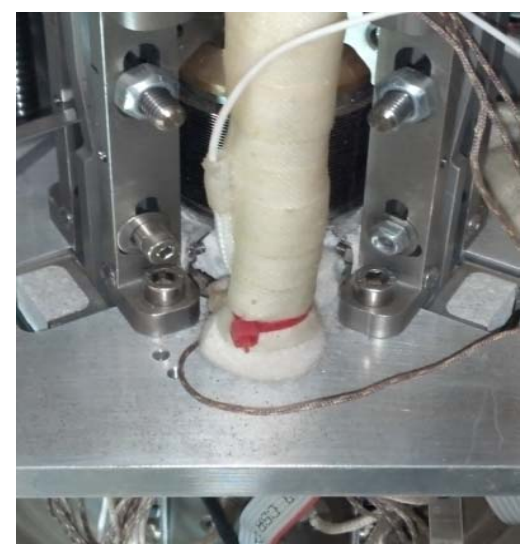

(b)

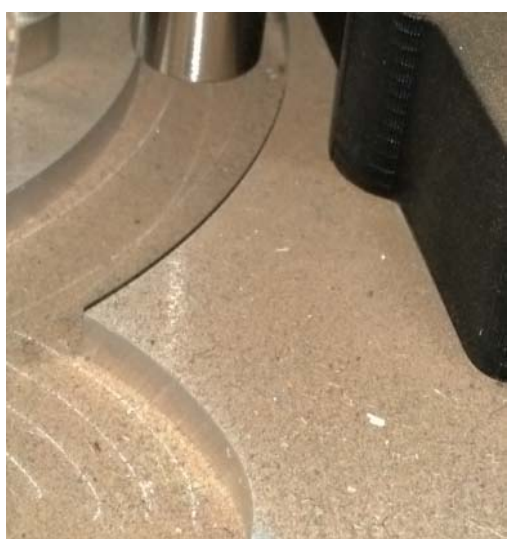

(c)

Figure 3. Dust mitigation (a)Polycarbonate panels fastened to OVEN FDU and taped at the seams

(a) Structural plate within upper portion of OVEN on the last day of field demonstration with no visible dust

(b) Structural plate within lower portion of OVEN on the last day of field demonstration with visible dust

\section{B. Carousel}

The purpose of the carousel is to move crucibles through the segment processing sequence by transporting them to the various mechanisms within the system. It is capable of rotating 360 degrees in either direction continuously, and can travel over $15 \mathrm{~mm}$ in the vertical axis. The conveyer plate is designed to hold and transport six crucibles. The crucibles are equally spaced 60 degrees apart and align with all mechanisms simultaneously. Three functional planes were established along the vertical axis for different circumstances. The rotation plane is used when transporting crucibles from one mechanism to another. Raising the carousel from the rotation plane to the activity plane will set a crucible at the proper height for a mechanism to interact with it. Raising the carousel to the stowage plane will stow the crucibles. This mechanism was heavily exposed to dust and performed extremely well throughout the duration of FDU testing. Fig. 4 illustrates the main components of the carousel used in the OVEN FDU. 
Despite the excellent performance of this mechanism, it will likely change during the next design iteration. A disadvantage of the carousel approach is the cross dependency it imposes on mechanisms around the system. The conveyor plate also creates a thermal bridge which would make it difficult to simulataneousely heat and cool crucibles in parallel. For these reasons, the carousel will likely be replaced with an arm that will be able to pick and place crucibles throughout the system in the next design iteration.

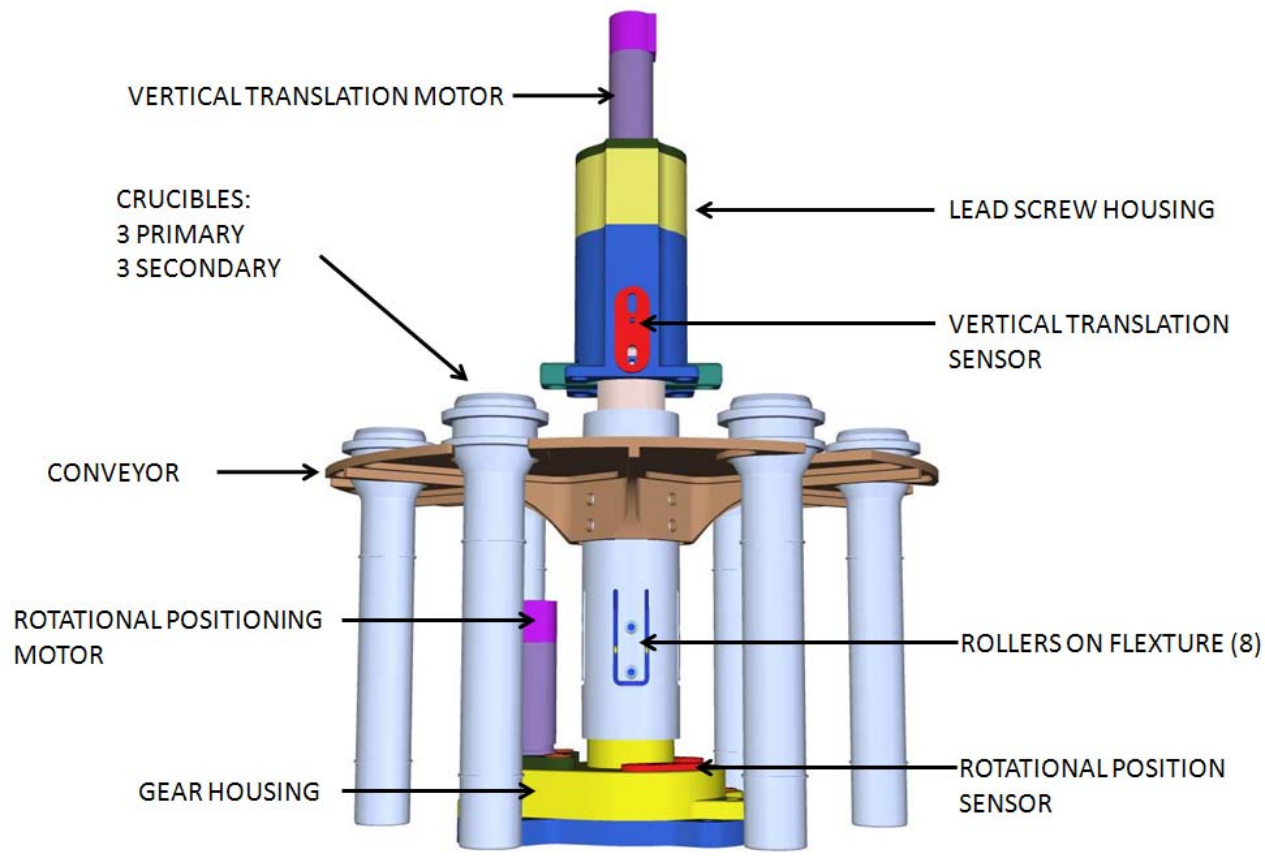

Figure 4. Carousel

\section{Stowage Snubbers}

The purpose of the crucible stowage subsystem is to hold the crucibles securely during launch and during rover traverses. The crucible stowage strategy in the FDU worked very well. The structure contained an array of spring loaded washers referred to as snubbers. Both the snubbers and crucibles were equally spaced 60 degrees apart along a common cylindrical plane. In order to stow the crucibles, the carousel was rotated to a position that aligned all six crucibles with all six snubbers. The carousel was then raised to an elevation defined as the stowage plane. The stowage plane was defined through testing as the elevation where the crucibles were held securely in place. The stowed configuration was used during shipping as well as roving operations. No problems related to stowage occurred throughout the duration of FDU hardware testing.

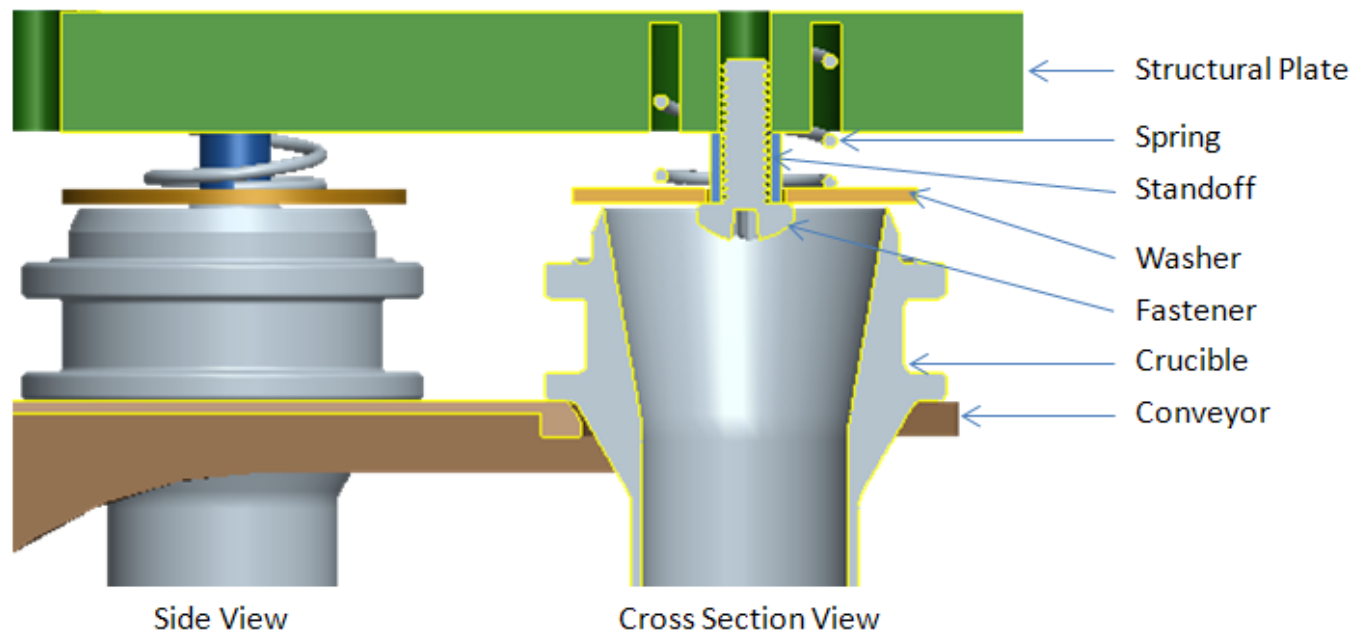

Figure 5. Crucible stowage

5

American Institute of Aeronautics and Astronautics 


\section{STR Interface}

The process of loading a new sample segment into the OVEN begins at the sample transfer receptacle (STR). The STR interface to the OVEN was coordinated so that a core segment can be loaded into an empty crucible located directly beneath the STR outlet. During the field demonstration, four unconsolidated segments of Hawaiian tephra were successfully transferred to the OVEN. Additional segments of JSC-1A simulant were also transferred during integrated tests at Kennedy Space Center. Solenoid tappers are located within the STR and the OVEN to promote the transfer of unconsolidated regolith. A barrier is used on the OVEN inlet to keep dust from migrating toward the sealing surface on a crucible during transfer. An inspection of the crucible sealing surface after integrated testing with the STR shows that with barrier was effective. The dust barrier serves an additional purpose of ensuring crucibles are properly aligned with all mechanisms. Early testing showed that misalignment with mechanisms could be problematic when the dust barrier was not in place. Installation of the dust barrier reduces the occurrence of misalignment to zero percent with all mechanisms in the system. The use of solenoid tappers as a means of promoting unconsolidated simulant flow is a method that was proven to be effective in previous RESOLVE reactor designs and was also effective during the FDU segment transfer process. ${ }^{6}$

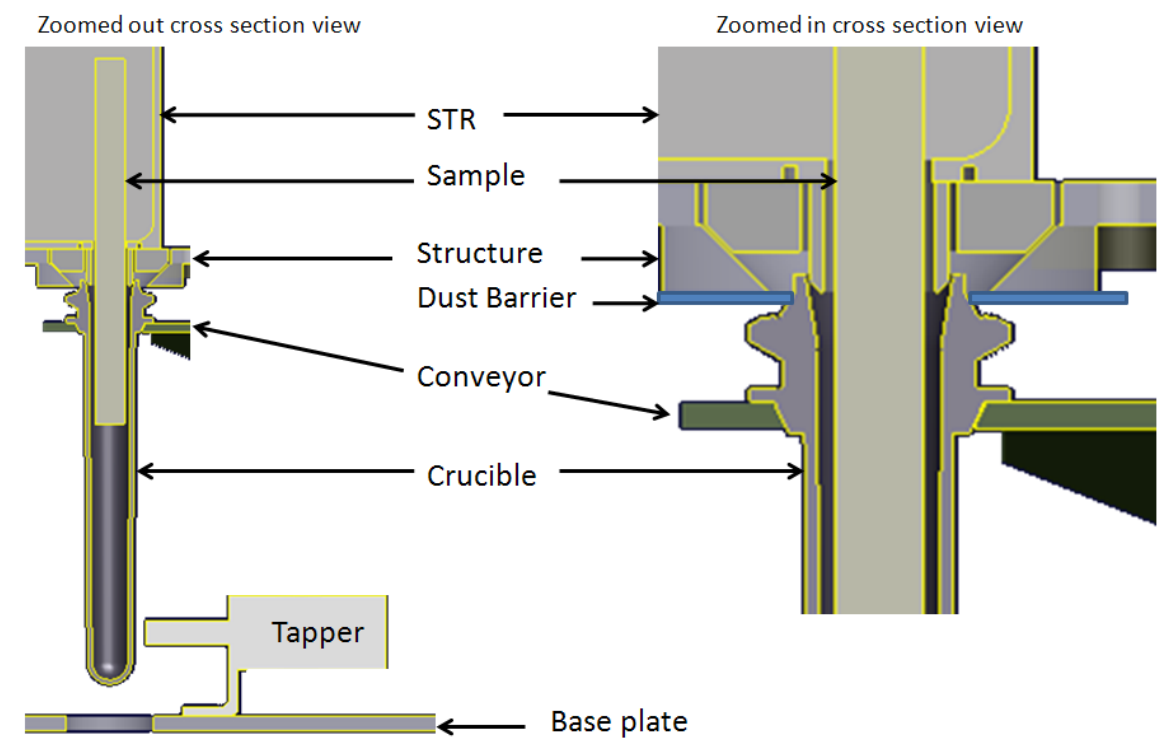

(a)

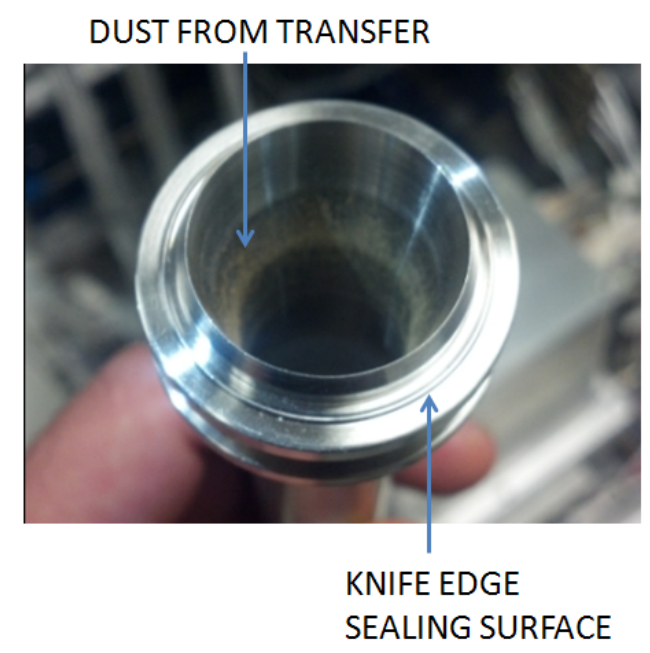

(b)

Figure 6. Segment loading (a)STR interface (b)Image of crucible after successful transfer

American Institute of Aeronautics and Astronautics 


\section{E. Weigh Station}

The weigh station is used to determine segment mass, as well as verify that a processed segment has been successfully expelled. An active crucible first engages the weigh station after receiving a new segment in order to determine the initial weight of the regolith. The crucible is also weighed before and after the regolith is discarded in order to determine that $95 \%$ of the sample has been expelled from the crucible. Crucibles are held in place by a latch and claw assembly that hangs from the load cell. The latch assembly is engaged when the carousel rises to the activity plane. When the carousel is lowered, the crucible hangs from the claws attached to the latch assembly. The latch assembly is disengaged when the carousel is raised to the activity plane again. The claws open as the carousel is lowered for the second time, and the crucible is released. Early design iterations showed unsteady readings due to friction between hanging components and static components. Removing material from the load cell structure allows the entire latch assembly to hang freely and no further issues were observed throughout the duration of FDU testing.

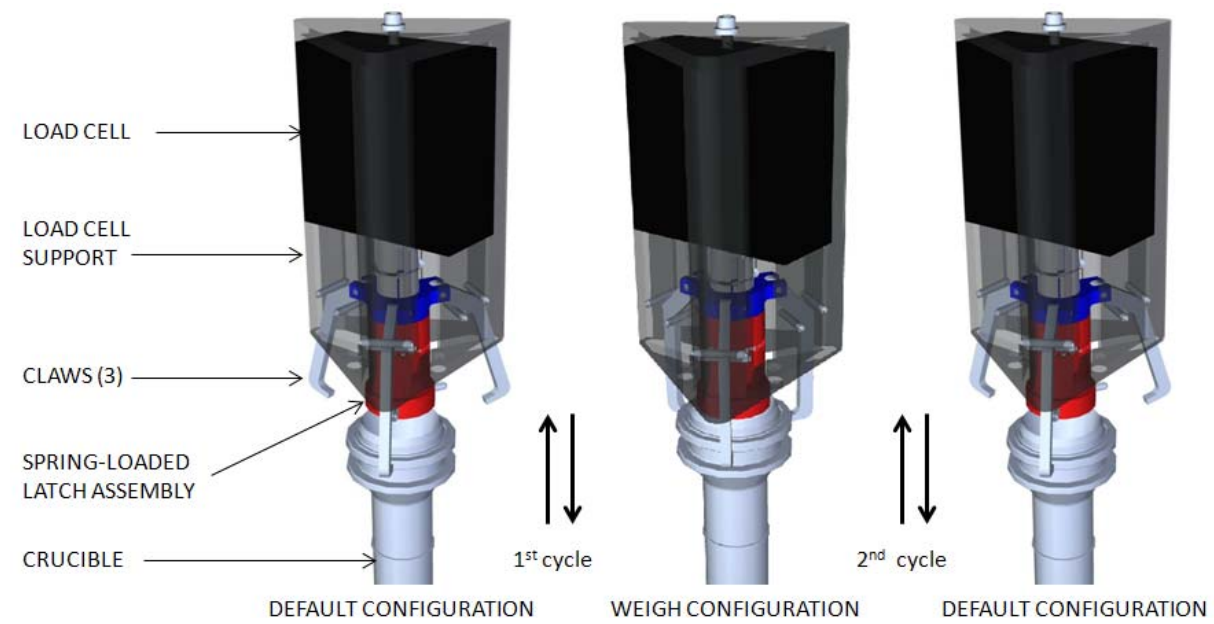

Figure 7. Weigh station

\section{F. Seal Dispenser}

Because the seals are exposed to dust and a temperature range of $200 \mathrm{~K}$ to $1173 \mathrm{~K}$, it was decided that the OVEN FDU would utilize single use seals to ensure that no volatiles were released during processing. The seal dispenser can hold up to 50 disposable seals. The seals are contained within the grooves of three ball screws supported by a gear assembly. As the central gear completes one revolution, a seal is dispensed from the bottom of the assembly onto the sealing surface of a crucible. Nickel $\mathrm{H}$-seals from Bostec were selected for extreme temperature use. Teflon seals were used for the majority of FDU testing in order to conserve the limited nickel seals. No problems were observed in this mechanism throughout the duration of FDU testing.

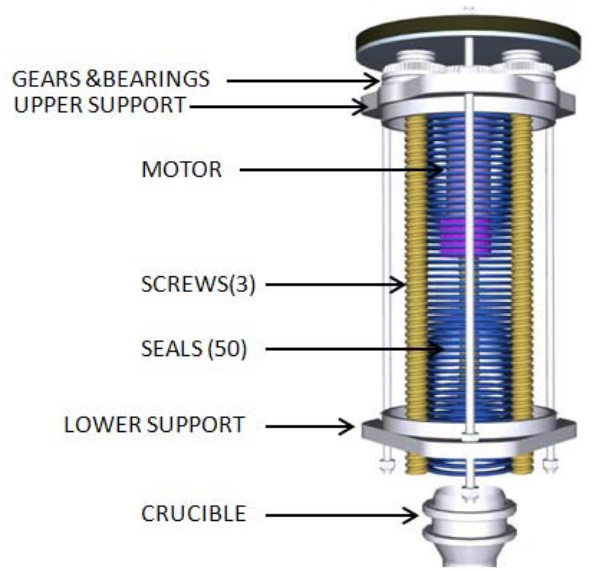

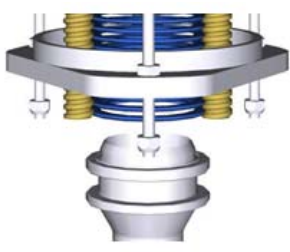

(a)

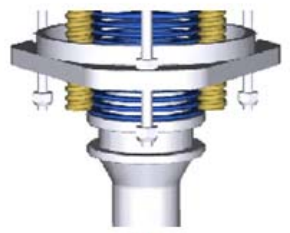

(c)

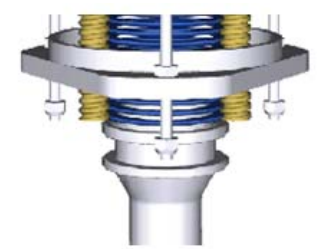

(b)

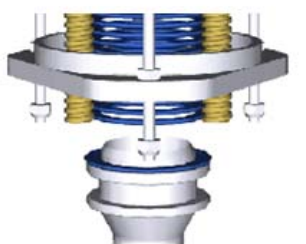

(d)

Figure 8. Seal dispenser (a)active crucible aligned with dispenser (b)carousel raises crucible to activity plane (c)ball screws revolve and release one seal (d)carousel lowers crucible with new seal in place 


\section{G. Capper}

The function of the capper is to generate the motion and force required to create a sealed volume within a crucible.The name "capper" can be misleading because it implies that caps are placed on the crucible. Caps are not used, but the motion of the mechanism is similar to that of a bottle capper as it grabs a crucible and raises it toward a fixed lid while applying hundreds of pounds of linear force. The motion is driven by three claws placed 120 degrees apart. The claws are attached to an ACME nut that translates along a lead screw driven by a worm gear and stepper motor. Both the crucible and fixed lid contain a knife edge that each penetrates 0.007 inches into a disposable nickel seal. As the crucible is removed, tabs on the claws insure that the seal is separated from the fixed lid. Motor faults were observed in this mechanism during integrated testing but were not consistant and were overcome by increasing motor power. Despite the faults, this mechanism completed many successful cycles without human intervention.
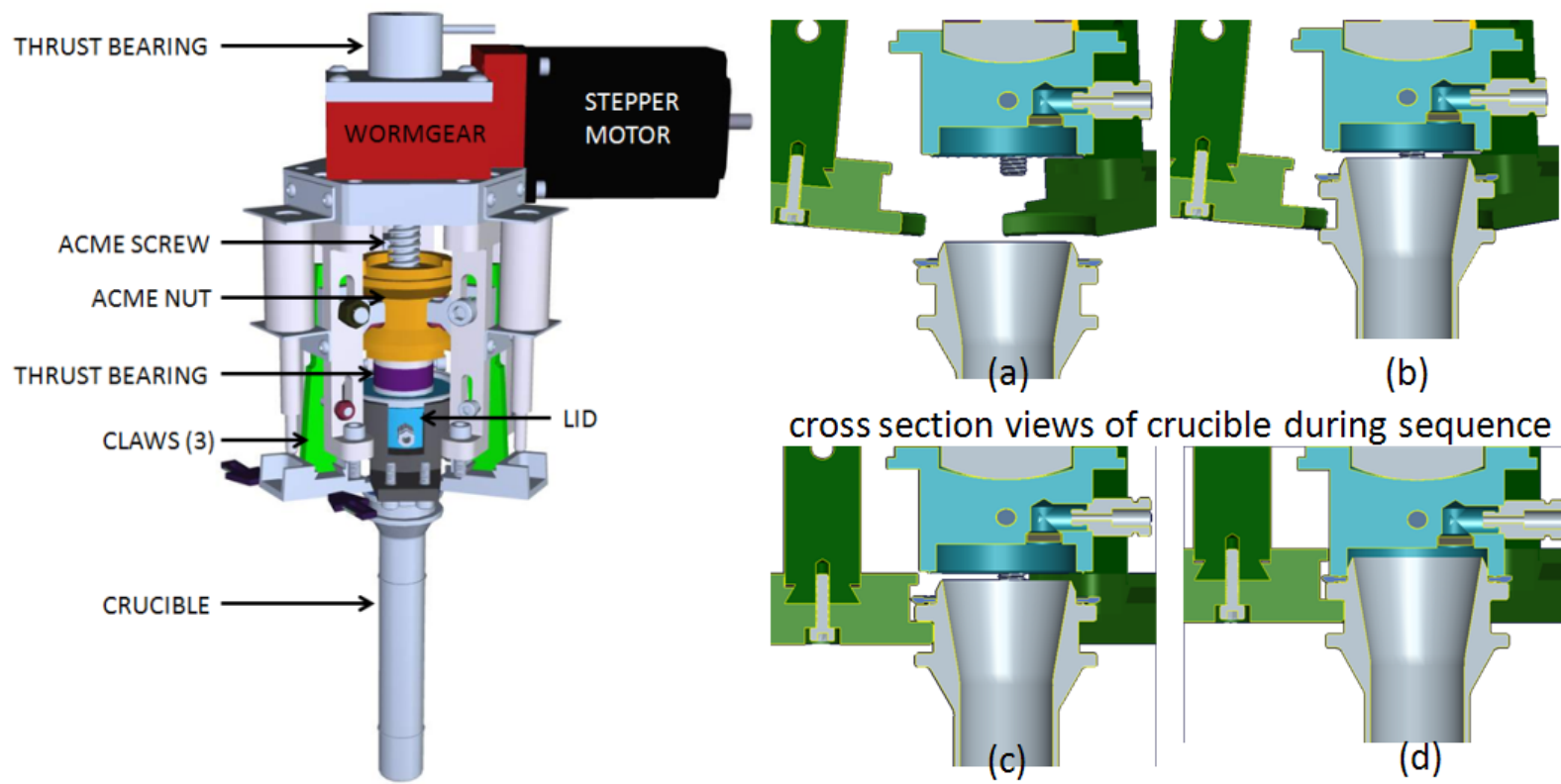

(b)

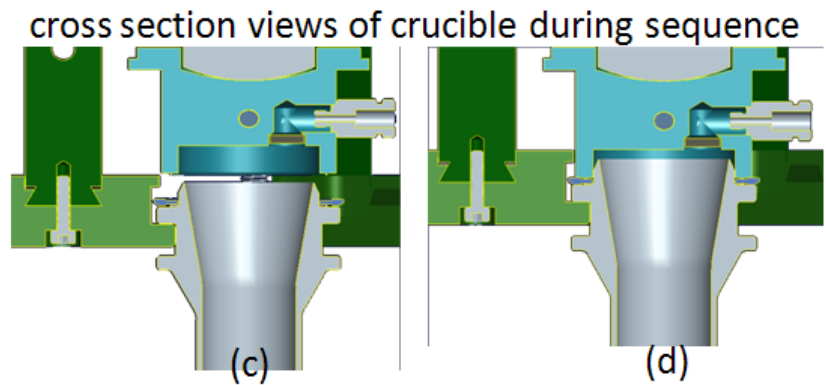

Figure 9. Capper (a)active crucible aligned with capper (b)carousel raises crucible to activity plane (c) claws grip crucible (d) crucible is raised by claws until seal is made

\section{H. Clamshell Heaters}

After a crucible has been successfully clamped to the capper mechanism, clamshell heaters are actuated to surround the crucible. The clamshell heaters and materials were selected that would allow a segment to be heated to $1173 \mathrm{~K}$. However, it was understood that this operating temperature would not be reached during a field demonstration without including a large amount of insulation. Only volatile analysis operations at $423 \mathrm{~K}$ were attempted during the field demonstration in order to keep the design and volume of the OVEN more representative of a lunar system. The heaters are built in to a clamshell mechanism that opens and closes around a sealed crucible. Each clamshell half included three zone heaters operating at 25W each. The purpose of using zone heaters is to reduce axial temperature gradient. Each zone heater is controlled by a K-type thermocouple near the heating element. Each zone also contains an additional thermocouple to collect a temperature data point near the outer diameter of the crucible. The clamshell mechanism was heavily exposed to dust and did not fail at any point during the demonstration. However, dust is believed to be the cause of some slippage during post-demonstration testing at KSC. After removing the dust, the mechanism completed the remaining cycles without incident.

The coiled nichrome heaters accomplished many heating cycles throughout the duration of FDU testing. Early testing at JSC determined that setting the heaters to $623 \mathrm{~K}$ would be sufficient to heat a crucible outer wall to $423 \mathrm{~K}$. The middle left heater was not used during the field demonstration because of control issues encountered during integrated testing at KSC. However, the five remaining heaters proved to be sufficient for heating samples during the field demonstration.

A different set of clamshell heaters are used for high temperature laboratory testing. The ceramic based heaters are more appropriate for reaching hydrogen reduction temperatures in a laboratory setting. They include insulation that is necessary when trying to achieve high temperatures within a room temperature atmosphere. 


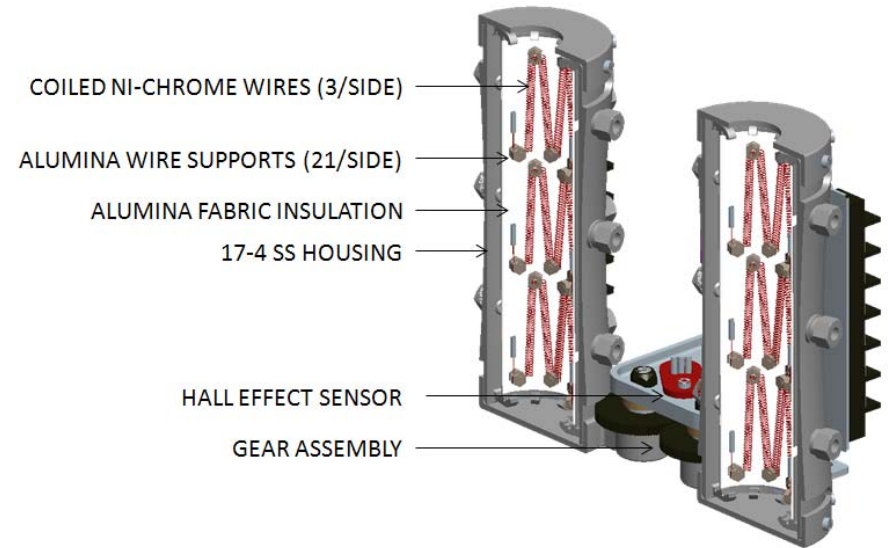

(a)

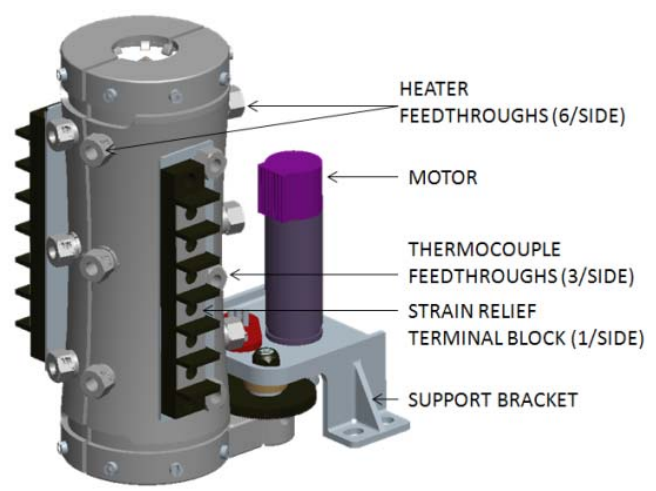

(b)

Figure 10. Clamshell Heater Assembly (a) open configuration (b)closed configuration

\section{Pressure Vessel}

The pressure vessel contains volatiles until they are transferred to the LAVA system for analysis and keeps hydrogen from escaping during the reduction process. The primary pressure vessel in the OVEN FDU consists of a crucible and lid. The crucible, lid and capper claws are all made of Inconel 625 to withstand temperatures up to 1223 $\mathrm{K}$. The crucible and lid were designed and tested to hold pressures up to 85 psig. Sintered metal filters were welded into the lid to prevent dust from flowing to components downstream. All pressurized components, tubing and manifolds were heated to $423 \mathrm{~K}$ during FDU operation. Pressurized components include a pressure transducer with embedded RTD and a relief valve set to 85 psig. During regolith oxygen extraction operations, the pressure within OVEN is set between 5-20 psig and hydrogen flows at a rate of 100-200 sccm.

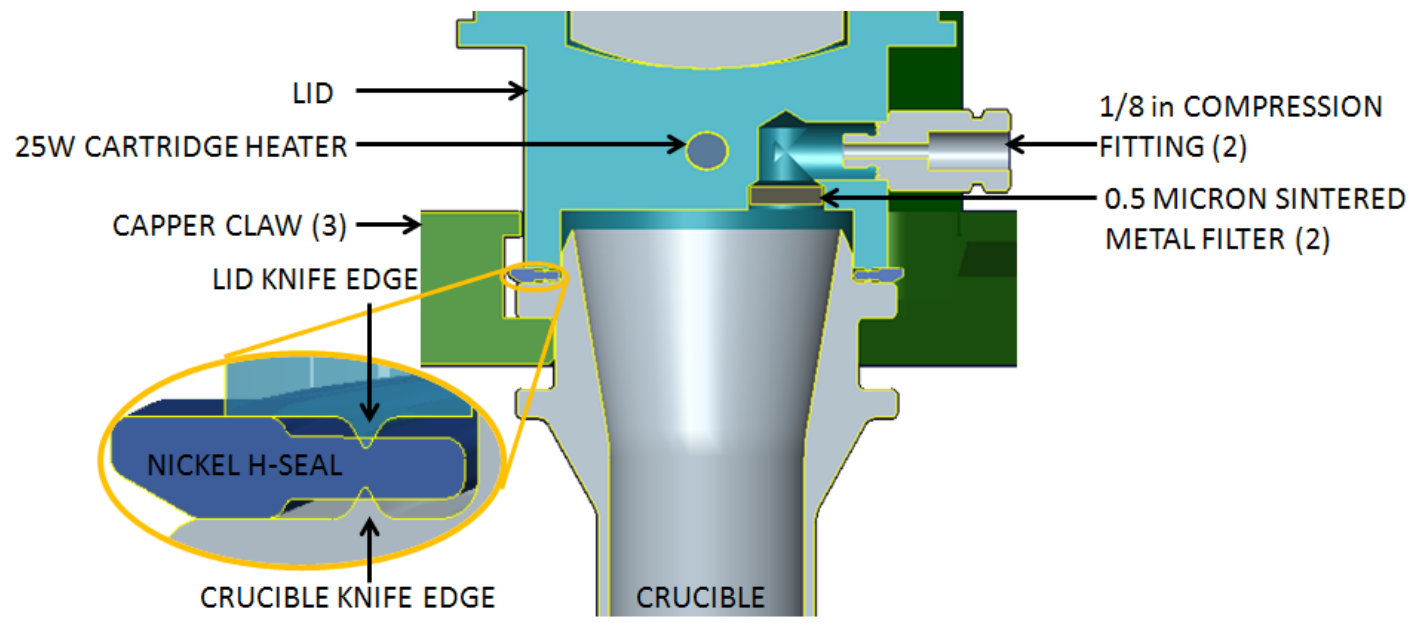

Figure 11. Cross section view of crucible and lid in sealed configuration

\section{J. Inverter}

The purpose of the inverter is to expel regolith from the crucible. It does this by rotating the crucible 180 degrees to an overturned position. A solenoid taps on the inverted crucible at a frequency of $8 \mathrm{~Hz}$ to assist in the removal of regolith. This mechanism worked well during initial laboratory testing at JSC, as well as integrated testing at KSC. A set screw on this mechanism became loose during shipping on the way to the field demonstration and was determined to be the cause of misalignment between the inverter claws and crucible. The misalignment caused a misplaced crucible during the last day of testing which required human intervention. The misalignment also affected the solenoid tapper and resulted in poor sample removal of the last two segments processed during the field demonstration. When FDU hardware returned to KSC, the set screw was tightened, and the inverter completed the remaining operation cycles without incident. 


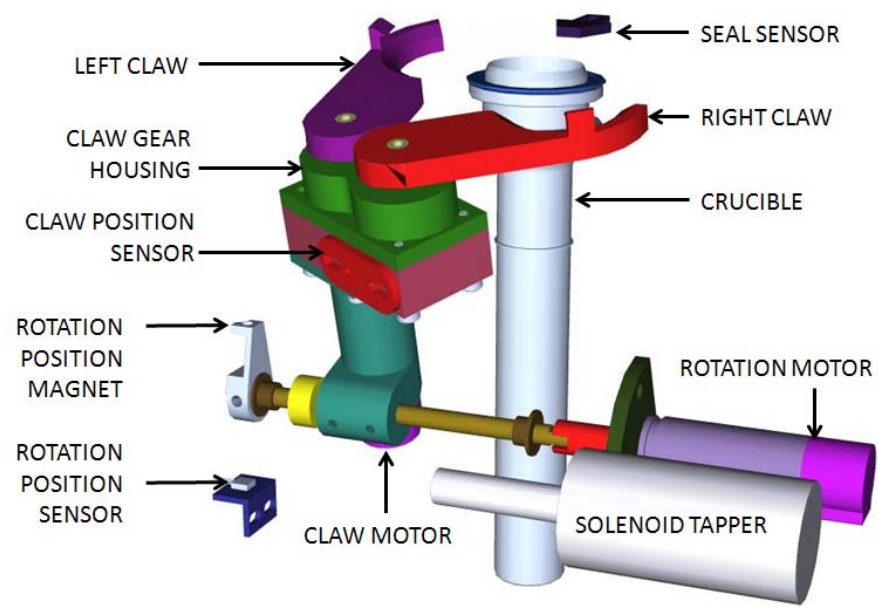

(a)

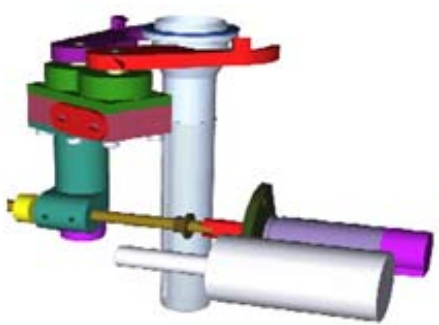

(b)

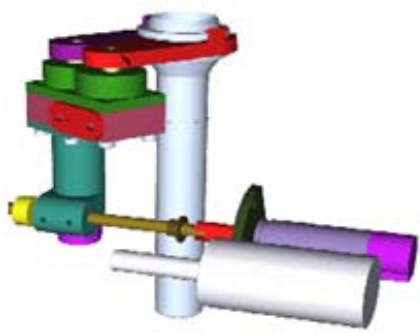

(e)

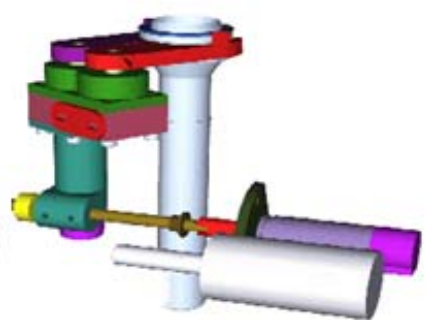

(c)

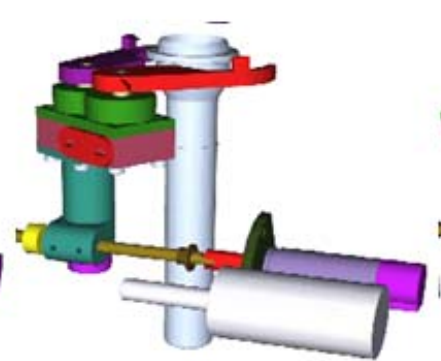

(f)

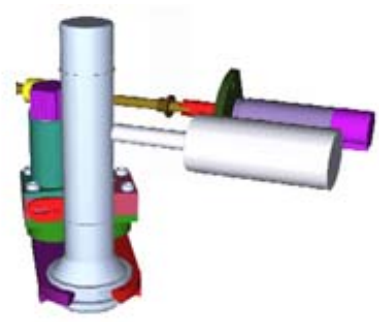

(d)

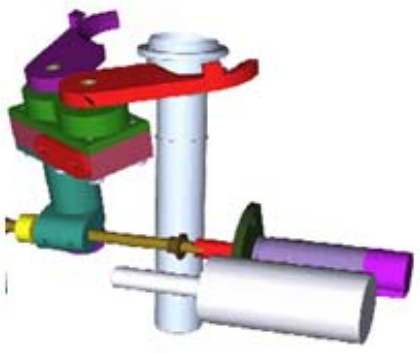

(g)

Figure 12. Inverter (a)initial configuration (b)claws remain open while rotating toward crucible (c)claws close to grip crucible (d)crucible is inverted, regolith and seal are removed (e)crucible is placed back on to carousel (f)claws open to release crucible ( $g$ )claws remain open while rotating away from crucible

\section{K. Chiller}

The purpose of the chiller is to ensure that the temperature of a crucible is lower than the temperature of an incoming segment during the loading process.The FDU chiller has the ability to cool crucibles below ambient temperature but was not used during the field demonstration as operation at low temperatures would have resulted in unwanted atmospheric water condensation. The FDU chiller is meant to serve as a placeholder for a cryocooler in future design iterations.

\section{Results and Discussion}

Several interim tests were performed throughout the development period of the OVEN FDU. Before the FDU OVEN design was finalized, proof-of-design concept tests were also performed Early tests include regolith mixing methods ${ }^{7}$, hydrogen reduction in a simulated crucible, volatiles release and regolith extraction. However, the results of these tests will not be discussed in this document as they resulted in the formation of FDU hardware. In this section, only the results of tests performed on FDU hardware will be discussed. 


\section{A. Seal Testing}

The pressure vessel of the OVEN is required to maintain a seal in a variety of extreme conditions. In order to perform hydrogen reduction, the pressure vessel must reach a temperature of at least $1173 \mathrm{~K}$. In a lunar environment, the pressure vessel must also form a seal at temperatures below $200 \mathrm{~K}$. In order to keep the volume of the pressure vessel to a minimum, the seal is exposed to both extremes. In addition, the presence of dust makes seal selection particularly challenging. In the early stages of FDU development, several seals were evaluated. Among the candidates were mesh-filled O-rings, spring-energized C-rings, V-rings, Omega-rings and H-seals. Most of the seals were made from high temperature alloys with high nickel content; some were coated with nickel or silver. All of the seals were capable of meeting the temperature requirements. Therefore dust, reusability, and crushing force were the criteria used in seal selection. The mesh-filled O-rings and spring-energized C-rings ranked highest for reusability, but required a high crushing force and were not suitable for use in the presence of dust. The V-rings and Omegarings were more suitable for use with dust but were observed to score sealing surfaces. Since the sealing surface would have to be built into the pressure vessel, this would mean the pressure vessels themselves could not be used more than once. The H-seal design requires the use of two knife edges that penetrate into both sides of a metal gasket. Although the seals themselves should be discarded after one use, the knife edges can be designed into the pressure vessel and used repeatedly. In addition, the H-seals required less crushing force than any other seal considered. The H-Seals were thus chosen to be used in the OVEN FDU and were subjected to a series of tests.

The goal of seal testing was to determine that the seals could meet a pressure decay requirement of $<1$ psi over 90 minutes for a variety of test conditions. The first test series was meant to observe resistance to dust. A large container was partially filled with JSC-1A simulant, and air was forced into the container using a fan in order to create a dust cloud. A nickel $\mathrm{H}$-seal was held inside of the dust cloud before being placed in a seal test fixture which was then pressurized to $\sim 60 \mathrm{psig}$. The next test series was designed to test the seal at low temperatures. The seal test fixture was chilled below $213 \mathrm{~K}$ using a free piston cooler and pressurized to $\sim 60 \mathrm{psig}$. The final test series was to meant to verify that the seal could hold pressure at temperatures in the range of $1173 \mathrm{~K}$. This test was accomplished using FDU hardware. A crucible was heated to $1173 \mathrm{~K}$ and pressurized to $38 \mathrm{psig}$ with a nickel seal installed. The $\mathrm{H}$-seals were tested at each test condition multiple times and were able to meet the pressure decay requirement in all cases.

\section{B. Hydrogen Reduction}

Hydrogen reduction of lunar material is a process that produces water vapor by removing oxygen from various forms of iron oxides found in the regolith. The advantage of this process as a method of ISRU is that it can be performed anywhere on the lunar surface and the reaction occurs below the melting point of lunar regolith, simplifying processing and removal ${ }^{8}$. However, the mineral content in lunar regolith is not homogenous across all of the lunar surface so the yield of the process varies depending on the mineral content at the location being explored. Since the destination for a mission with the RESOLVE payload is driven by the presence of water, not reducible material, the hydrogen reduction process to be demonstrated by the OVEN system is not considered critical to the success of a water prospecting mission.

In order to show that the OVEN FDU system is capable of demonstrating the hydrogen reduction process, two OVEN systems were built. One system was integrated into the RESOLVE system and participated in the field demonstration. The other system remained at the Johnson Space Center so that high temperature testing could be accomplished in parallel. The high temperature system utilized more insulation than the field unit, which allowed it to reach the temperatures required without being placed in a vacuum chamber. High temperature data was collected using two configurations. In the first configuration, illustrated by Fig. 13a, a multi-point thermocouple was inserted into a crucible filled with 38 grams of JSC-1a lunar regolith simulant. The thermocouple was inserted from the top. The bottom of the crucible and clamshell heaters were well insulated, but the top of the crucible was exposed to room temperature atmosphere. The multi-point thermocouple provided seven data points spaced one inch apart. The second configuration, illustrated by Fig. 13b, was used during hydrogen reduction tests. In the second configuration, the multi-point TC was inserted through the bottom of the crucible. Both configurations utilized the same clamshell heaters. The temperatures of the six clamshell heaters were given by TCs 101-106. The temperatures along the multipoint-thermocouple were given by TCs 115-121. An axial temperature gradient of several hundred degrees was observed in both cases. This was due to heat losses to the surrounding atmosphere at the ends of the crucible. The lower temperatures observed have an impact on the yield of the hydrogen reduction process. Despite the temperature gradient, the FDU design was able to demonstrate the extraction of oxygen from JSC -1a simulant. Thermal analysis and testing in a vacuum chamber will be used to determine a design configuration that can achieve maximum yield in a relevant environment. However, because hydrogen reduction is for demonstration only, any attempt to increase the yield of this process must not interfere with the ability to extract water, and must have a minimal mass impact. 


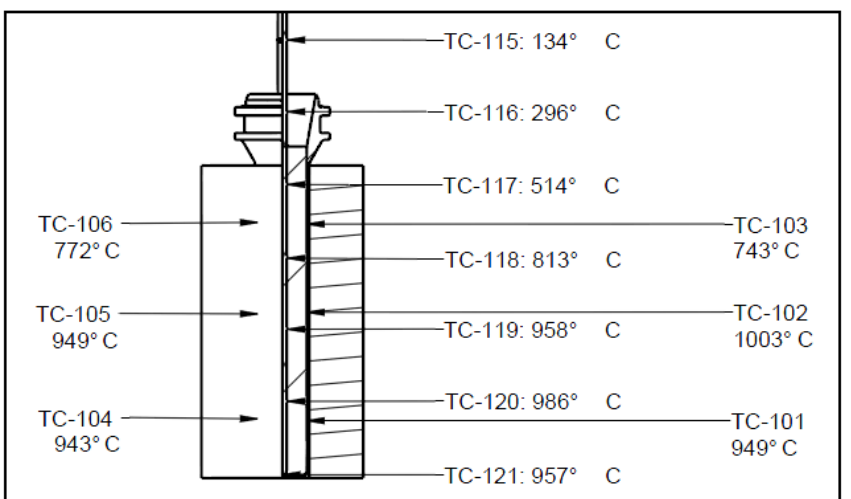

(a)

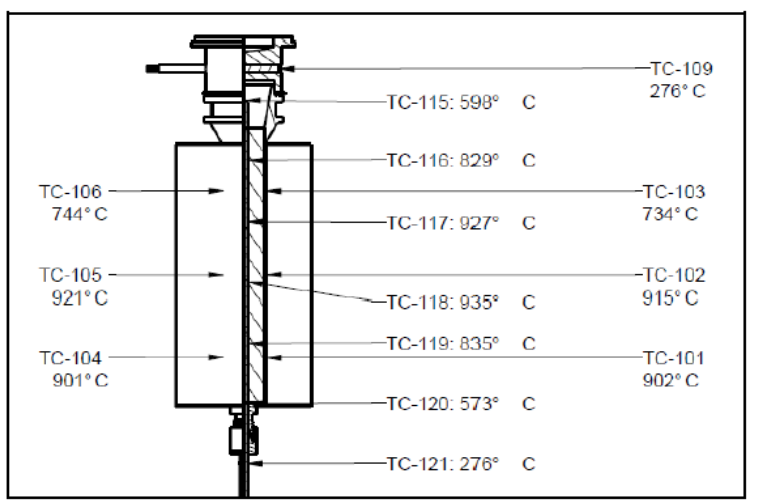

(b)

Figure 13. High temperature data collection (a)multi-point thermocouple inserted from top (b)multi-point thermocouple inserted from bottom

Several hydrogen reduction tests were performed using configuration (b) illustrated in Fig.13, all of which demonstrated the ability to produce water vapor. Water production was determined by weighing a desiccant dryer downstream of the OVEN before and after the hydrogen reduction process. Fig. 14 illustrates the typical results of a hydrogen reduction test. Table 1 shows a summary of hydrogen reduction results. These results will be used to determine the feasibility of performing a water droplet demonstration on the lunar surface. Further tests will be performed with different simulants containing smaller amounts of reducible material. Additional testing will also involve placing the OVEN in a vacuum chamber during reduction operations. Other gases such as carbon monoxide and methane will be evaluated as reducing agents in future tests.

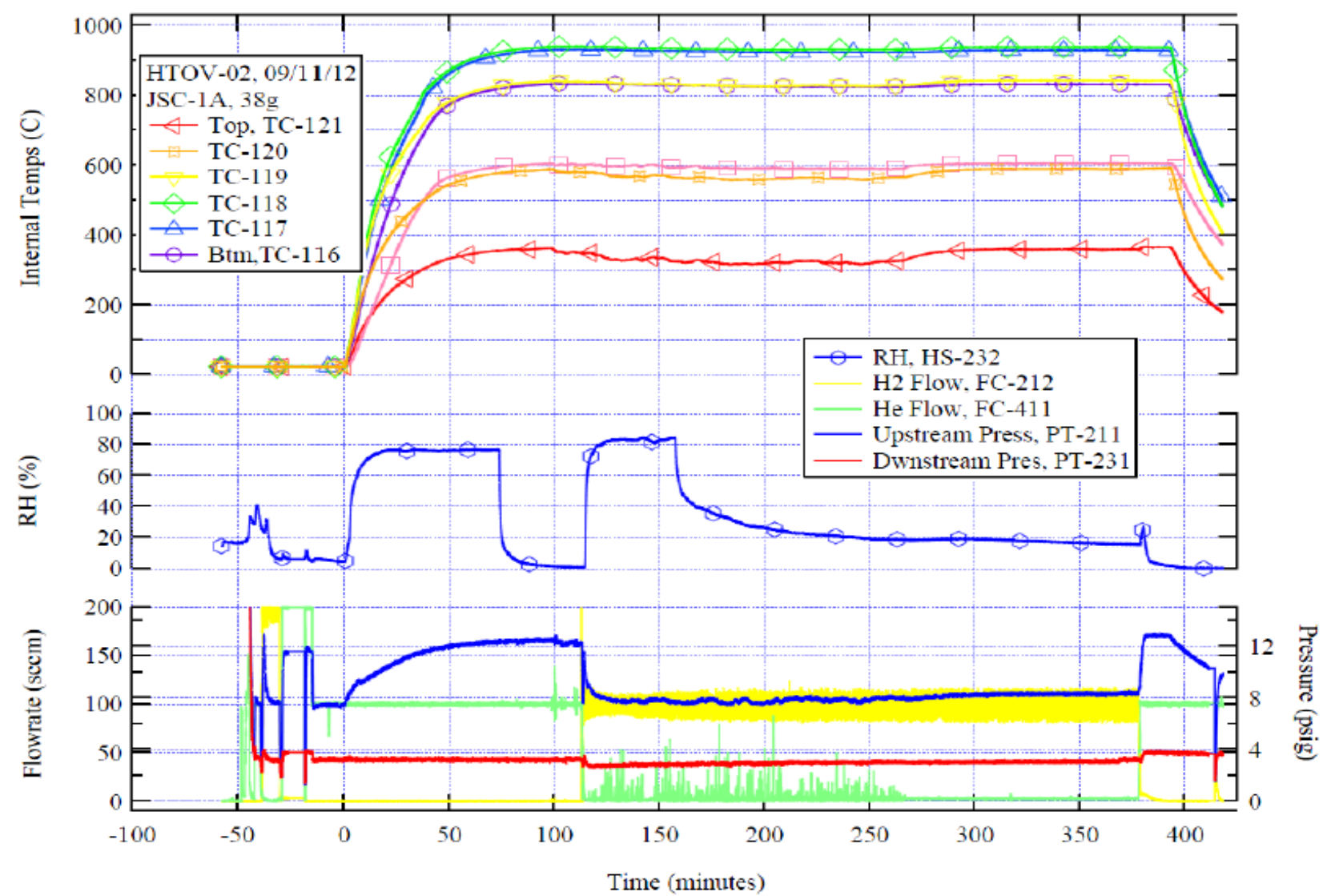

Figure 14. Example of hydrogen reduction test results 


\begin{tabular}{|l|l|l|l|l|}
\hline test \# & water produced & duration & sample mass & flow rate \\
\hline & grams H20 & hours & grams JSC1-A & sccm \\
\hline 2 & 0.241 & 4.5 & 38 & 100 \\
\hline 3 & 0.228 & 4.5 & 38 & 100 \\
\hline 4 & 0.160 & 4.5 & 19 & 100 \\
\hline 5 & 0.167 & 4.5 & 19 & 100 \\
\hline 6 & 0.238 & 4.5 & 38 & pulse \\
\hline 7 & 0.134 & 2 & 19 & 200 \\
\hline 8 & 0.190 & 4.5 & 19 & 200 \\
\hline 9 & 0.197 & 5 & 19 & 200 \\
\hline
\end{tabular}

Table 1. Results of hydrogen reduction tests for OVEN FDU design

\section{Conclusion}

The design approach used to meet the functional requirements for the OVEN system in the RESOLVE FDU should work well for future design iterations. All of the mechanisms showed the capability of being used for multiple cycles successfully. The issues that were encountered during testing could be easily diagnosed and addressed. The separation of functions into multiple components allowed for issues to be resolved without disturbing or disassembling other parts of the system. This design should be able to accommodate the introduction of segments below $175 \mathrm{~K}$ without major modifications by replacing the FDU chiller with a cryocooling station. The field demonstration was an excellent proving ground for dust exposure. The impact of dust exposure to the mechanisms within the OVEN was not as severe as anticipated during the July 2012 field demonstration.

\section{Acknowledgments}

This work was made possible by the NASA Advanced Exploration Systems organization through the RESOLVE project, and by the Johnson Space Center Propulsion and Power Division. The authors are grateful for the outstanding work of the entire RESOLVE team and the JSC Energy Systems Test Area for making the integration and operation of this subsystem successful.

\section{References}

${ }^{1}$ Smith, P.H., et. al, “H $\mathrm{H}_{2} 0$ at the Phoenix Landing Site,” Science, Vol. 325, No. 58, July 2009, pp. 58-61.

${ }^{2}$ Colaprete, A., et. al, "Detection of Water in the LCROSS Ejecta Plume,” Science, Vol. 330, No. 463, Oct. 2010, pp. 463468.

${ }^{3}$ Larson, W, Picard, M, Quinn, J, et. al, "NASA’s Lunar Polar Ice Prospector, RESOLVE: Mission Rehearsal in Apollo Valley”, 63 ${ }^{\text {rd }}$ International Astronautical Congress, Naples, Italy, October, 2012.

${ }^{4}$ Andreas, E.L., "New estimates for the sublimation rate for ice on the Moon,” Icarus, Vol. 186, No. 1, Jan. 2007, pp. 24-30.

${ }^{5}$ McKay, D. S., Allen, C.C., "Hydrogen reduction of lunar materials for oxygen extraction on the moon," AIAA $34^{\text {th }}$ Aerospace Sciences Meeting and Exhibit, Reno, NV, Jan. 15-18, 1996

${ }^{6}$ Kleinhenz, J., Yuan, Z., Sacksteder, K., Caruso, J., "Development of a Reactor for the Extraction of Oxygen and Volatiles from Lunar Regolith," $47^{\text {th }}$ AIAA Aerospace Sciences Meeting Including The New Horizons Forum and Aerospace Expostion, Orlando, FL, Jan. 2009.

${ }^{7}$ Nayagam, V., Berger, G.M., Sacksteder, K.R., Paz, A., "Experimental Measurements of heat Transfer through a Lunar Regolith Simulant in a Vibro-Fluidized Reactor Oven,” AIAA 50 "Aerospace Sciences Meeting, Nashville, TN, Jan. 2012.

${ }^{8}$ Cooper, B.I., "Sintering of Lunar and Simulant Glass," AIAA Space Technology and Application International Forum, CP969, Albuquerque, NM, 2008. 